\title{
DIE VERWANDTSCHAFTSBEZIEHUNGEN ZWISCHEN MOLLUSKEN UND ANNELIDEN
}

VON

\author{
H. F. NIERSTRASZ \\ Utrecht.
}

In dem Sammelbande „Die Kultur der Gegenwart” hat HeIDer der Phylogenie der Mollusken einen Abschnitt gewidmet (8, pag. 504). Es ist immer eine Freude, Artikel dieses hervorragenden und klaren Forschers zu lesen; eine eben so grosse Freude macht es denn auch ihn zu bekämpfen.

Der Inhalt des genannten Abschnittes ist zwar nicht für Zoologen geschrieben worden. HEIDER deutet aber die Punkte, um welche sich die ganze Sache handelt, so genau und richtig an, dass die ganze Frage in knapper und deutlicher Fassung vorliegt. Das Resultat dieser Erörterungen ist dieses, dass die anatomischen und entwicklungsgeschichtlichen Tatsachen mehr für eine Herleitung der Mollusken von Anneliden sprechen sollen als von Turbellarien. In die zoologische Sprache übersetzt wäre dies so zu verstehen: Waren die Vorfahren der Mollusken näher mit denen der Anneliden als mit denen der Turbellarien verwandt?; oder: Zeigt der Bauplan der Mollusken eine grössere Aehnlichkeit mit dem der Anneliden oder mit dem der Turbellarien? Ueber solche Fragen ist viel gestritten worden und wird wohl immer "gestritten werden, denn es handelt sich nur um Auffassungen, nicht um kontrollierbare Tatsachen. Und alles hängt davon ab, wie man sich die „Construction” der ganzen Tierwelt vorstellt.

\section{ALLGEMEINER BAU}

Vergleicht man den Bauplan der Mollusken und Anneliden, so lässt sich nicht leugnen, dass es nur sehr wenige Punkte von Uebereinstimmung gibt. Es lassen sich beinah keine zwei Tiertypen nennen, deren Bauplan so grundverschieden ist. Eigentlich kann man wohl sagen, dass es kaum Uebereinstimmung gibt. Dies erkennt auch HEIDER selbst, wenn er sagt: „In diesem ganzen Kreise (d. h. der Würmer und der wurmähnlichen Formen) vermissen wir die geringsten Spuren jener Organe, durch welche die Mollusken gekennzeichnet sind” (8, pag. 507), und weiter: „Habituell sind natürlich Ringelwürmer und Mollusken sehr verschieden" (8, pag. 509). HEIDER versucht aber dennoch $z$ zu einem Compromis zu kommen. Wie nun nach ihm das Blutgefässsystem der Mollusken auf das der Ánneliden zurückgeführt werden könnte, ist mir ein Rätsel; ebenso rätselhaft ist es mir, wie Heider den Zustand der Lamellibranchier mit ihrem vom Rectum „durchbohrten” Herzen zurückführt auf den Bau der Blutgefässe der Anneliden, vor allem deswegen, weil sein Schema B (7, pag. 283) dem wirklichen Zustand bei den Lamellibranchiaten gar nicht entspricht. Ueberdies entsteht diese "Durchbohrung" des Herzens auf ganz andere Weise, nämlich dadurch, dass sich das Pericard um das Rectum herumschlägt und ventral von diesem verwächst. Und schliesslich kommt ein durchbohrtes Pericard bei weitem nicht bei allen Blattkiemern vor; es muss vielmehr ein freies, dorsal vom Rectum gelegenes Herz als primitiv angesehen werden (15, pag. 94). - Der Bau des Nervensystems der Mollusken lässt sich ebensowenig zurückbringen auf den der Anneliden, wohl aber auf einen indifferenten Zustand, von welchem sich beide Systeme herleiten lassen; diesen Zustand findet man bei den Turbellarien. 


\section{DAS CÖLOM}

Die Hauptbetrachtungen bei solchen Ueberlegungen liefert immer der Bau- des Cöloms, dieses rätselhaften, nicht genau definierbaren Begriffes. Mit vielen anderen nimmt HEIDER an, dass bei den Mollusken das Cölom reduciert ist, ohne aber diese Meinung näher zu begründen (7, pag. 276; 8, pag. 510). Die Möglichkeit hiervon wird nicht von mir bezweifelt; ein Hinweisen aber auf die Arthropoden, bei welchen Formen eine analoge Reduction stattfinden soll, ist nicht zulässig, weil bei den Arthropoden das Cölom gut entwickelt und zwar segmentiert auftritt und man dieses Cölom bei der Embryonalentwicklung Schritt für Schritt sich reducieren sieht. Weder aus der Entwicklungsgeschichte, noch aus dem Bau der Mollusken aber ist eine einzige Tatsache bekannt, welche auf eine ähnliche Reduction hinweisen soll. Zwar könnte man sich berufen auf die Cephalopoden, welche eine grosse secundäre Leibeshöhle zeigen; auf diesen Punkt werde ich später zurückkommen. Dies wird auch von HEIDER zugegeben, wenn er schreibt: „Weder im Körperbau, noch in der Ontogenie der Mollusken sind deutliche, unzweifelhafte Spuren zu erkennen, welche auf eine ehemalige Segmentierung ihrer Ahnenformen hinweisen" (8, pag. 511). Erstaunlich ist es denn auch, dass HeIDER hierauf etwas weiter folgen lässt: „Wenn sonach bei gewissen Anneliden die Metamerie fast völlig in Verlust geraten kann, so werden wir aus ihrem Fehlen bei den Mollusken keinen Gegengrund gegen die Annahme einer Abstammung dieser Gruppe von Ringelwürmern erblicken können.” Hieraus ergibt 'sich, dass HeIDER im Inneren seiner Seele das Verschwinden der Metamerie bei den Mollusken doch annimmt. Wenn aber keine einzige Tatsache weder aus der Anatomie, noch aus der Ontogenie bekannt ist, aus welcher sich eine Reduction der Segmentierung nachweisen liesse, so ist es m. E. nicht erlaubt anzunehmen, dass es jemals eine Segmentierung gegeben hat.

Es gibt überdies noch andere Gründe für die Auffassung. Was ist doch die Bedeutung des gegliederten Cöloms? Ueber diese Frage ist relativ. wenig geschrieben worden. Wohl hat man sich manchmal den Kopf zerbrochen mit der Frage: „In welcher Weise ist das Cölom entstanden und hat sich dieser Raum „in Stücke” gebrochen”? Die wohlbekannte Pseudometamerie-theorie von LANG, die Cormentheorie, vor allem von HATSCHEK propagiert, die Cyclomerentheorie von SEDGwick, die Nephrocöltheorie von FAUSSEK, alle diesen versuchen das Entstehen einer secundären Leibeshöhle zu erklären. Im Wesen des Cyclomeren- und der Cormentheorie liegt die Notwendigkeit einer Metamerie aufgeschlossen; in den beiden anderen ist dies auch der Fall, aber das "Warum" bleibt bei diesen verborgen. Ich will hier nicht weiter diese Theorien besprechen, schliesse mich aber mit HEIDER der Ableitung der Metamerie von den Pseudometamerien an; diese würde „unter den umordnenden Einfluss der Lebensweise” geschehen sein (8, pag. 494). „Durch terminales Längenwachstum wurde zunächst eine ungegliederte langgestreckte Stammform erreicht, worauf der Gesamtkörper durch eine Umordnung der einzelnen Organe gleichzeitig in eine grössere Anzahl von Segmenten zerfällt wurde. Diese Annahme stützt sich auf den Gedanken, dass bei der seitlich schlängelnden Bewegung des Körpers und bei der durch die zunehmende Differenzierung bedingten Starrheit der Gewebe die Ausbildung alternierender Regionen grösserer und geringerer Beweglichkeit von beträchtlichem Vorteil für das Individuum war und eine weitere Längenausdehnung des Körpers ermöglicht. Es würde dann die erste Ursache für das Auftreten der metameren Gliederung in der Bewegungsweise und in mechanischen Verhältnissen zu suchen sein" (8, pag. 495). In der Tat scheint hierin der Kern der Wahrheit zu liegen; diese Auffassung sagt dennoch nichts vom Auftreten der wahren Metamerie, d. h. vom Auftreten von sich wiederholenden, mit „Wasser” gefüllten Räumen, deren Bedeutung nicht erläutert wird, denn eine „Ausbildung alternierender Regionen grösserer und geringerer Beweglichkeit” führt nicht zu einer Metamerie; die wahre Metamerie setzt das Vorhandensein einer Cölomhöhle voraus. - Nun ist es bemerkenswert, dass eine wahre unverfälschte Metamerie nur bei den Anneliden vorkommt, d. h. bei Tieren mit langem gestrecktem Körper, welche schlängelnde Bewegungen ausführen und eine grabende (im weitesten Sinne des Wortes genommen) Lebensweise führen. Dies bringt auf den Gedanken, dass es zwischen Körperform, Segmentierung und Lebensweise einen innigen Verband gibt und zwar zwischen diesen drei Factoren zusammen. Soll ein langgestrecktes, wurmförmiges Tier im Stande sein, seine eigentümlichen schlängelnden Bewegungen $z u$ vollziehen, so ist eine erste Bedingung, dass alle Organe gleichmässig über den Körper sich verbreiten; die sehr regelmässige Contractionen machen ein voll- 
kommenes Zusammenspiel eines regelmässig gebauten Muskelsystems, aus Längs-, Ring- und schief gestellten. Muskeln' notwendig. Aber ebenso notwendig ist es, dass ein solches Tier eine feste Achse besitzt, welche im Stande ist, dem Körper eine gewisse Steifheit zu geben, den Druck im Körper regelmässig zu verteilen und einen Gegendruck auf die sich zusammenziehenden Muskeln auszuüben. Solch eine Achse besitzen die Würmer in der Cölomflüssigkeit; sie haben eine steife und doch biegsame Wasserachse. Aber diese Achse würde niemals etwas erreichen, wenn sie ununterbrochen vom Vorder- bis zum Hinterende des Tieres durchginge, denn bei Contraction einer willkürlichen Stelle des Körpers würde das Wasser proximad und distad ausweichen, wodurch die Regelmässigkeit unterbrochen werden sollte. Deshalb ist die Wasserachse in eine Zahl von kleinen Achsen geteilt, welche voneinander durch wasserundurchlässige Schotten getrennt sind. Jetzt ist jeder Teil der Wasserachse im Stande, für einen kleinen Teil des Körpers seine Wirkung auszuüben; die Dissepimente verhindern ein Ausweichen des Wassers in proximale und distale Richtung. Nur ein ganz vollkommenes Zusammenspiel der regelmässig gestellten Muskeln mit den Wasserkämmerchen ermöglicht die vollkommen schlängelnde Bewegung. Wenn denn auch nur etwas in diesem System fehlt, so fällt die typische Wurmbewegung weg. Hierfür geben die Nemertinen und Balanoglossus Beweise; beide Typen sind wurmförmig; keiner aber ist im Stande, schnelle, kräftige, schlängelnde Bewegungen auszuüben. Bei beiden sehen wir zwar eine regelmässige Stellung der Organe, notwendig für Windungen, Knickungen und Biegungen; weiter aber kommt es durch das Fehlen der kleinen Wasserachsen nicht. Der echte Wurmhabitus wird nie erreicht. Ebensowenig bringen die Nematoden es weiter als ein eigenartiges "Hin- und Herpendeln der Körperenden in der Medianebene”. (17; pag. 89). - Zudem aber spielt die Cölomflüssigkeit der Anneliden eine grosse Rolle beim Graben; WolterECK hat schon hierauf hingewiesen (21, pag. 302). Ein gutes Beispiel hierfür liefert Lumbricus, dessen Grabbewegungen ermöglicht werden durch Muskelcontractionen und Wasserbewegung im Vorderteil des Körpers. Wenn es keine Dissepimente gäbe, so würden die Muskeln des Vorderendes allein nicht im Stande sein, die für das Graben nötige maschinelle Wirkung zu liefern. Meines Erachtens hat das segmentierte Cölom mit seinem wässerigen Inhalt ursprünglich nur Bedeutung für die Bewegung. In dieser Hinsicht ist es ziemlich gleichgültig, in welcher Weise es entsteht. Es kommt mir aber vor, dass ein näherer Zusammenhang zwischen Gonaden und Cölom sehr wohl zu verstehen ist. Ist es doch eine bekannte Sache, dass bei erwachsenen Tieren es beinah immer einen innigen Verband gibt zwischen Gonaden und Cölomepithelium. Bei Anneliden werden denn auch die Geschlechtsprodukte in der Cölomwand gebildet; die "Geschlechtsorgane" "flottieren” in Wasser, was für sie bei den schlängelnden Bewegungen des Körpers die sicherste Stelle ist, weil das Wasser am besten im Stande ist, verschiedene Druckverhältnisse allmählig $z u$ verteilen. - Es gibt natürlich eine grosse Menge von Fällen, welche weiteren Erläuterungen bedürfen, z. B. das Localisieren der Geschlechtsdrüsen; die Gelegenheit fehlt mir aber, um an dieser Stelle näher hierauf einzugehen. Jedenfalls erlangt die Gonocöltheorie durch diesen engern Zusammenhang einen höheren. Wert. Jeder besondere Fall bei den Anneliden wird mit Rücksicht auf die Lebensweise genau untersucht werden müssen; z. B. wird die Beschaffenheit des Mediums, in welchem gegraben wird, einen belangreichen Faktor bilden bei der Beurteilung der Segmentierung; die Reduction dieser letzteren, z. B. bei Formen, welche im Meeressand leben, ist meistens sehr gut zu verstehen. Dass weiter bei Sipunculus keine Dissepimente vorhanden sind, kann nicht verwundern, weil dieses Tier eine beinah sedentäre Lebensweise führt, welche sich im Bau abspiegelt (Verlegung des Anus nach vorn, u.s.w.); in der Jugend sind Spuren von Metamerie nachzuweisen, wenigstens nach Gerould, was aber von Spengel verneint wird (16, pag. 104). Ebensowenig ist es fremd, dass die Segmentierung verschwindet, sobald eine andere Bewegungsweise auftritt; so bei den Arthropoden, bei welchen Formen ein steifes Exoskelett und gegliederte Extremitäten dem Mechanismus der Bewegung vollkommen andere Gesetze vorschrieben; eine Wasserachse wäre hier ein Widersinn. Andererseits ist es eigenartig, dass bei festsitzenden Tieren niemals Spuren von echter Segmentierung wahrgenommen werden, wohl aber grössere oder kleinere Cölomsäcke, z. B. bei Brachiopoden, Bryozoen, Pterobranchier und Sipunculus. Welche Bedeutung dieser Zustand hat, kann ich nicht angeben. Das Cölom hat aber eine noch viel weitere Bedeutung als oben angeben wurde, wenigstens secundär (Wasserregulierung in Verband mit dem Auftreten eines Blutgefässsystems, Excretion), sodass in concreten Fällen die Bedeutung von vornherein nicht festgestellt werden kann; zum grössten Teil aber 
gehören diese Fragen zum Gebiet der Physiologie. - Dass schliesslich die echte Segmentierung bei Vertebraten verschwindet, lässt sich leicht verstehen, weil diese ein eigenes Achsensystem im Notochord besitzen und deshalb die Wasserachse mit den Wasserkämmerchen entbehren können.

Vergleicht man jetzt die Mollusken mit den Anneliden, so findet man total andere Verhältnisse. Auch die Mollusken "grąben" und kriechen. Der Fuss ist das Organ, mit welchem die Bewegungen statt finden; die Blutflüssigkeit ist das Agens, welches Schwellung und Contraction der Bewegungsorgane reguliert; der Körper selbst aber ist beim Kriechen und Graben passiv. Deshalb gibt es denn auch kein Cölom bei den Mollusken, jedenfalls kein Cölom im Sinne der Anneliden. Deshalb auch keine Spur von Metamerie. Man könnte nun bemerken, dass bei den Amphineuren eine gewisse Pseudometamerie vorhanden ist, aus welcher sich jedoch eine echte Metamerie entwickeln könnte. Dies ist aber nur scheinbar. Die Solenogastren haben zwar grösstenteils die Wurmform erlangt; von wurmförmigen Bewegungen ist aber keine Rede; sie bewegen den Körper zwar schlängelnd, aber äusserst langsam; eine gewisse Regelmässigkeit der Stellung der inneren Organe wird deshalb vorhanden sein; eine Wasserachse aber ist für diese trägen, steifen Tiere ganz überflüssig. Diese Tiere „werden es niemals zu segmentierten Tieren bringen." Dasselbe gilt für die Chitonen; von einer verschwundenen Segmentierung ist keine Spur vorhanden; nur zeigen gewisse Organe eine Pseudometamerie, welche verständlich ist für aufrollbare, in der Brandung lebende Tiere (z. B. Schalen und Kiemen; c. f. die Cyclobranchier, welche ausser den. Kiemen keine Spur von Pseudometamerie zeigen, sich aber auch nicht aufrollen können). Meines Erachtens ist es nicht zulässig, das sogenannte Cölom der Mollusken mit dem der Anneliden zu vergleichen. Dieses "Cölom", das Pericard, ist klein und nicht reduziert, wie die Ontogenie lehrt; es liegt hinten im Körper, über dem Darm und steht ursprünglich mit den Gonaden in Zusammenhang, wobei es durch zwei Gänge ausmündet. - Vor einigen Jahren habe ich versucht zu zeigen, dass dieses "Cölom" durch Vereinigung der Geschlechtsgänge entstanden ist, welche darauf wieder auseinander gehen; so entsteht in der Mitte dieser Gänge ein Eiersack (13). Diesen einfachen Zustand findet man wirklich bei einigen einfachen Solenogastren beinah genau so wieder. Erst später, wenn sich durch Localisation der Atmung im Analraum Kiemen bilden und ein regelmässiger Blutstrom von den Kiemen zu der dorsal vom Darm gelegenen Aorta notwendig wird, entwickelt sich an der dorsalen Wand des Eiersackes ein Herz und wird das Säckchen zum Pericard. Neuerdings hat ODHNER sich für diese Auffassung ausgesprochen (14, pag. 76). Daher die doppelte Function des Pericards: es functioniert sowohl als dieses wie als Eiersäckchen; die Eier selbst zeigen eigenartige Formen, weil sie mit der beweglichen Herzwand in Zusammenstoss geraten. Factisch ist der Zustand so bei den Solenogastren, und diese Tiere werden doch von den meisten Forschern für die primitivsten, jedenfalls für sehr primitive Mollusken gehalten. Die doppelte Function des „Pericards” wird erst aufgehoben, wenn die Gonaden eigene Ausführungsgänge erlangen und sich deshalb vom Pericard absondern; letzteres füllt sich mit Wasser, welches gewisse Bedeutung für die Herzbewegungen besitzt und zu gleicher Zeit für die Wasserregulierung des ganzen Körpers, während beide Gänge, welche das Pericard mit der Aussenwelt verbinden, excretorische Function erlangen; sie sind jetzt zu „Cölomoducten" geworden, wenn man wenigstens dem Pericard den Namen Cölom zukennen will. Meines Erachtens aber ist die Bedeutung eine ganz andere als bei den Anneliden; man darf beide Zustände nicht direct miteinander vergleichen. Dass das Pericard sich in kleine Hohlräume teilen und in dieser Weise eine Segmentierung auftreten sollte, würde natürlich keinen Sinn haben. Und will man sich noch auf ontogenetische Beweise berufen, so ist ebenfalls ein grosser Unterschied vorhanden. Bei den Anneliden entsteht das segmentierte Cölom durch Erweiterung der pseudometamer gestellten Gonadenhöhle (Gonocöltheorie); bei den Mollusken dagegen entsteht das ungegliederte "Cölom” durch Vereinigung der Ausführungsgänge der Geschlechtsdrüsen, welche in der Einzahl vorhanden sind. - Was nun die Cephalopoden betrifft, so sind hier wohl grosse Höhlen vorhanden, welche man mit dem Cölom vergleichen könnte. Ich glaube aber nicht, dass die Cephalopoden primitive Formen sind; es kommt mir vor, dass die pelagische Lebensweise dieser Tiere, welche doch zu einer Klasse mit ausgesprochener benthonischer Lebensweise gehören, secundär ist. Ich neige zu der Ansicht, dass die Cephalopoden ursprünglich festsitzende Tiere waren. Ihre Neigung zu radiärer Symmetrie, ihre eigenartige Drehung, welche ganz anders verläuft als bei den Gastropoden, ihr concentriertes Nervensystem, ihr "geschlossenes" Blutgefässsytem, u. s. w. sprechen m. E. für diese Meinung. Wie ich schon auf 
pag. 35 bemerkte, kommt ein grosses Cölom eben bei festsitzenden Formen vor. Dass Abel neuerdings in tiefgehenden Studien eine Meinung ausspricht, welche mit meiner in Widerspruch steht, kann meine Auffassung nicht ändern (1). Später hoffe ich die Gelegenheit zu haben diese Sache näher auseinanderzusetzen.

\section{TROCHOPHORA UND PROVELIGER}

HEIDER legt weiter den Nachdruck auf die Uebereinstimmung, welche in der Entwicklung der Anneliden und Mollusken bestehen soll (8, pag. 508). Es handelt sich um den Bau der Larven und die Cell-lineage.

Der Bau der Larven wird von HEIDER ausführlich behandelt. Sowohl die Anhänger wie die Gegner der Trochophora-theorie sind zahlreich; diese Sache ist so oft besprochen worden, dass es schwer fällt, noch etwas Neues zu finden. So wie überall kommt es auch hier an auf die Auffassung über das Entstehen der mehrzelligen Organismen und ihre ursprüngliche Lebensweise. Dass diese Lebensweise ursprünglich eine pelagische war, kann ich nicht einsehen. Das Leben ist auf dem Boden des Meeres entstanden! Eine solche Auffassung lässt sich natürlich nicht beweisen; persönliche Sympathien spielen hierbei natürlich eine grosse Rolle. Die Erwägung aber, dass Protoplasma schwerer ist als Meereswasser, spricht für meine Meinung. Das pelagische Milieu ist kein natürliches Milieu; kein Tier, ein- oder mehrzellig, ist von Haus aus dem pelagischen Leben angepasst. Dies lehren die pelagischen Tiere selbst; alle zeigen spezielle Anpassungen, welche sie für das pelagische Milieu fähig machen und welche alle daraufhin gerichtet sind das spezifische Gewicht zu verringern, oder jedenfalls den Tieren eine grössere Treibmöglichkeit zu garantieren, z. B: das Auftreten von Vergrösserung der Oberfläche, von Luftblasen, Oeltropfen, Treibapparaten, u. s. w. Kommen solche Anpassungen nicht vor, dann müssen die pelagischen Tiere durch fortwährende Schwimmbewegungen das Herabsinken verhindern. Ein natürliches Milieu kann man dies kaum nennen! Die Trochophora mit ihrer „Kugel”form, ihrem sehr einfachen Bau und dem Schwimmapparat passt in diese Vorstellung. Aber auch dieser einfache Bau weist auf die Richtigkeit meiner Auffassung hin. Die ursprünglichen „mehrzelligen” Tiere waren nicht so ganz einfach gebaut, wie man es wohl vorstellt. Auch hier gehen die Meinungen weit auseinander. Wie muss man sich das Auftreten von „mehrzelligen” Individuen vorstellen? Man könnte sie auffassen als Kolonien von „einzelligen Individuen”, z. B. von Flagellaten; (9, pag. 92). HeIDER spricht von einem "Aggregat gleichartiger Protozoenindividuen" (8, pag. 453). Mir kommt dies irrtümlich vor. Es bestehen über diese Sache fehlerhafte Meinungen, welche daraus resultieren, dass man die Protisten für einzellige Organismen hält. Man findet diese Meinung bei HeIDer (7, pag. 184); weiter bei LANG und DOFLEIN, wie ClifFoRd DoBELl in einem trefflichen Artikel hervorhebt (3, pag. 275). Letztgenannter Forscher hat in 1911 mit Recht den Unterscheid zwischen dem omnipotenten Protistenorganismus und den beschränkt potenten Zellen der Metazoen klar auseinandergesetzt. Destomehr muss man sich darüber wundern, dass DofLEIN in seinem bekannten Lehrbuch von 1916 noch immer schreiben kann: „In ihrem (d. h. der Protozoen) gesamten Aufbau entsprechen die typischen Protozoen nur einer jener Einheiten, aus denen sich der Körper der vielzelligen Tiere wie aus vielen Bausteinen aufbaut; sie bestehen aus einer einzigen Zelle" (4, pag. 3). HARTMANN meint in 1912: „Die Protozoen sind einzellige Organismen.... Die einzelnen Individuen besitzen nur den Formwert einer einzigen Zelle" (5, pag. 1126), welche Meinung er 1919 noch nicht ganz aufgeben kann (6, pag. 89). Es kommt nun nicht an auf die genaue Definition des Begriffes „Zelle”, sondern auf die Tatsache, dass der Protist nur mit dem ganzen Metazoon und nicht mit dessen absonderlichen "Bausteinen” verglichen werden kann, wohl aber mit der befruchteten Eizelle des Metazoons. Es geht nicht an, dieses als eine Kolonie von Protisten anzusehen; das Metazoon ist eine Zellfamilie, welche durch successive Teilung der omnipotenten "Eizelle” entstanden ist. Aggregieren oder Zusammenfliessen von Protisten $\mathrm{zu}$ bleibenden mehrzelligen Individuen ist in der Natur niemals beobachtet worden. Die einfachste „Kolonie” Gonium ist keine Kolonie, sondern eine Protistenfamilie, denn es entsteht aus successiven Teilungen des befruchteten Isogametes; alle Glieder sind omnipotent, und es gibt noch keine Arbeitsteilung. Viel weiter geht Pandorina, bei welcher Form dieselbe Familie schon Makro- und Mikrogameten liefert, die Arbeitsteilung setzt ein, die Potenzen gehen auseinander, sie ist nicht mehr eine Protistenfamilie; noch weiter geht Eudorina mit ihren männlichen und weiblichen Familien, und zuletzt 
kommen Pleodorina und Volvox. Die Omnipotenz der Eizelle löst sich allmählich in die beschränkten Potenzen der "Bausteine” auf. Dieser Prozess lässt sich demnach phylogenetisch so gut wie möglich verfolgen; überdies tritt er bei der ontogenetischen Entwicklung von allen höheren Metazoen deutlich zum Vorschein. Ein höheres Tier würde eine Kolonie darstellen, wenn viele oder mehrere befruchtete Eier sich miteinander vereinigten um zusammen das Individuum zu bilden. Das geschieht aber niemals; jedes Tier ist eine wahre Zellfamilie. - Abgesehen hiervon ist es nun interessant nachzugehen, in welcher Weise die Entwicklung zu mehrzelligen Individuen weiter statt findet. Die einfachsten und am meisten ursprünglich gebauten Organismen werden wohl aus einer unregelmässigen Anhäufung von omnipotenten Elementen zusammengesetzt gewesen sein, so wie man dies jetzt noch in der Morula und bei Gonium findet. Das heisst: eine solche Anhäufung zeigt Componenten an der Oberfläche, welche ein gewisse Zahl von centralen Componenten umschliessen. Geht nun die Entwicklung solcher Anhäufungen weiter und werden diese grösser, so werden die. inneren Componenten bald in ganz andere Lebensverhältnisse geraten als die äusseren bedeckenden. Letztere dienen für Schutz, Bewegung, Atmung und Ernährung; die inneren dagegen sind mehr passiv und dienen z. B. für die Fortpflanzung und zur Stütze. Dies wird soweit gehen, bis die Lebensbedingungen der inneren Componenten derart werden, dass ihr Leben bedroht wird durch Sauerstoff- und Ernährungsmangel, abgeschlossen wie sie von ihrem Milieu sind. Um diesem Uebelstande abzuhelfen sind verschiedene Heilmittel vorhanden. Die Anhäufungen flachen sich ab zu Platten oder es tritt eine Einstülpung auf, welche das Wasser in das Innere führt. Geschieht dieses, so entsteht eine Form, welche stark einer Gastrula ähnelt, abgesehen vom äusseren Habitus, was weniger belangreich ist, und abgesehen von der Tatsache, dass solch einfach gebautes Tier besteht aus einer äusseren deckenden Schicht, aus einer inneren Schicht, welche das Meereswasser ins Innere führt und mit diesem Sauerstoff und Nahrung, und natürlich aus Gruppen von Componenten zwischen diesen beiden Schichten, welche das Ganze auffüllen. In dieser Weise entsteht auch eine Arbeitsteilung: die äussere Schicht wird „Haut”, die innere „Darm”; die zwischenliegenden Componenten gewähren eine Stütze. Mit dieser Arbeitsteilung geht die Omnipotenz der Componenten verloren; die Potenzen verteilen sich über die Compotenten; die Zellen treten auf. Nur die Geschlechtselemente bisweilen auch noch andere - bleiben omnipotent. Die Fortpflanzungs, zellen” werden sich wohl dort befinden, wo sie, als das kostbarste Element, die beste Aussicht für ihr Fortbestehen haben, nämlich in der Wand oder in der Nähe des "Darmes”. Die ursprünglich vielzelligen Tiere setzten sich deshalb aus drei Zellcomplexen zusammen, wenn man will aus drei „Keimblättern”. Diese Herleitung kommt mir logischer vor als jene, nach welcher die Blastula und Gastrula natürliche Stadien in der Entwicklung der Vielzelligen repräsentieren sollten. Uebrigens können Blastula und Gastrula auch nicht direct mit mehrzelligen Tieren verglichen werden; sie sind nur mehrzellige vorübergehende Stadien in der Entwicklung von vielen Mehrzelligen. Sie sind viel zu einfach und viel zu regelmässig gebaut um ein Bild der ursprünglich ganz unregelmässigen Zellfamilien zu geben. Viel besser, glaube ich, könnte man beide als Anpassungen an das pelagische Leben betrachten. Denn ebenso wie das ursprüngliche Leben benthonisch war, haben auch die Vorstufen von Blastula und Gastrula dieses Leben geführt. Dass eine solche Anpassung statt gefunden hat, kann kaum befremden; diese befördert die Verbreitung der Art und gibt ausserdem den Larven Gelegenheit sich besser zu ernähren - auch mit Sauerstoff -, als es auf dem Boden möglich war. Deshalb zeigen Blastula und Gastrula, infolge der Anpassungen, welche das pelagische Leben fordert, einen einfacheren Bau; es tritt die Kugelform auf; die mittleren Zellen verschwinden, d. h. werden in ihrer Anlage gehemmt und ihre Entwicklung wird auf später verschoben; sie liegen ruhig aufgeborgen im inneren oder äusseren Blatt. Und erst später treten auch die mittleren Zellmassen zum Vorschein, nicht als etwas Neues, sondern als etwas Altes, was nur durch die Bedingungen einer besonderen Lebensweise in seiner Entwicklung gehemmt worden war. Solange nun das pelagische Leben dauert, ebenso lang behält das.junge, sich entwickelnde Tier oder die Larve ihren „pelagischen” Habitus. Demnach wird bei den Anneliden die Trochophora ebenso Merkmale dieser Anpassung zeigen müssen. Das tut sie denn auch in der Tat; der ganze Habitus mit seinen Wimperkränzen, die Sparsamkeit der Gewebe zwischen Darm- und Hautblatt weisen hierauf hin. Die Trochophora ist kein „junger Wurm”; sie repräsentiert nur ein vorübergehendes Larvenstadium mit besonderen Anpassungen an eine Lebensweise, welche weder den ursprünglichen mehrzelligen Lebewesen, noch den Anneliden zukommt. Und sie wandelt sich auch nicht in den jungen 
Wurm um, sondern letzterer wächst an oder in der Trochophora in einer bestimmten Wachstumszone, benutzt dabei einige ihrer Organe und Gewebe und wirft schliesslich alles, was ihr das Treiben möglich machte $a b$, denn erst jetzt fängt die endgültige Lebensweise an. Ganz analoge Prozesse findet man bei anderen Tierklassen, bei welchen im Grunde des Wesens dasselbe geschieht, z. B. bei den Nemertinen, den Echinodermen, Phoronis, Balanoglossus, u. s. w. Für mich ist die Trochophora eine Anpassungsform, derer Entwicklung ich mir - soweit möglich - erklären und vorstellen kann. Unlogisch aber kommt es mir vor, sie als Stammform zu deuten; überdies sehe ich keinen Grund, warum eine solche pelagische Stammform ihre pelagische Lebensweise aufgeben und mit einer benthonischen wechseln sollte, ebensowenig wie dies möglich wäre. Denn in Wirklichkeit sinkt die Trochophora erst, nachdem der junge Wurm an oder in ihr gewachsen, also schon gebildet ist, ganz einfach durch die Schwerkraft dem Boden zu. Nicht die Trochophora sinkt, um am Boden in ein benthonisches Geschöpf sich umzuändern, sondern sie wird, nach Bildung dieses letzteren, ganz einfach mechanisch nach unten gezogen. Demnach ist es m. E. plausibel, dass die Trochophora keine Urform gewesen ist, sondern dass die Urform benthonisch gelebt haben muss. Es muss doch der auf diese Sache sich beziehende Passus in der übrigens schönen Arbeit von WOLTERECK als blasse Phantasie aufgefasst werden (21, pag. 300)!

Wie steht es nun mit der "Trochophora". der Mollusken? Es lässt sich nicht leugnen, dass äusserlich sowie innerlich yiele Punkte von Uebereinstimmung mit der Anneliden-trochophora bestehen. Hierüber aber muss man sich nicht allzusehr wundern. Für die Mollusken gilt natürlich dasselbe, was von der Entwicklung im Allgemeinen gesagt worden ist; auch bei diesen Tieren entsteht der dem pelagischen Leben angepasste Veliger - oder besser gesagt Proveliger, wie NAEFF mit Nachdruck hervorhebt (12, pag. 381) - aus einem ebenso dem pelagischen Leben angepassten, einfachen Keim, der Gastrula. Proveliger und Trochophora werden sich wohl in vielen Hinsichten ähneln müssen, weil beide sehr einfach gebaute, demselben Leben adaptierte Formen sind, z. B. im Habitus, im Vorkommen von Wimperkränzen, von denen aber die Trochophora mehrere besitzt. Am frappantesten ist wohl, dass bei beiden eine orale Scheitelplatte mit den Ganglien vorkommt; inwieweit dieses Sinnes- und Bewegungsorgan direct mit der Lebensweise in Verband steht, lässt sich ohne Weiteres nicht bestimmen. Dass bei beiden Formen der Darm etwa denselben Verlauf hat, ist verständlich, ebenso das Vorkommen einer Mutterzelle des "mittleren Keimblattes", dessen Entwicklung in Folge der Lebensweise unterdrückt worden ist. - Daneben aber gibt es auch grosse Unterschiede. Vorerst fehlen beim Proveliger, soweit mir bekannt ist, alle radiär von der Scheitelplatte verlaufenden Nerven der Trochophora. Und dann ist der weitere Gang der Entwicklung ein ganz anderer. Der Wurm wächst an oder in der Trochophora, wirft aber einen grossen Teil dieser Larvenform ab. Beim Proveliger aber ist dies ganz anders: er verwandelt sich selber in einen Mollusk. Es werden zwar einige Teile, wie die Wimperkränze, beim Uebergang der pelagischen in die benthonische Lebensweise abgeworfen, im grossen Ganzen aber wird der Proveliger selber zu einem Veliger und dieser zu einem Mollusk. Hier betrachten wir also kein An- oder Einwachsen des jungen Tieres, sondern ein sich Verwandeln. Der Proveliger spielt auch hier eine vorübergehende Rolle, aber in ganz andrem Sinn wie bei der Trochophora. Dieser Unterschied lässt sich nur verstehen, wenn man beide Typen auf die Turbellarien zurückführt; einerseits führte der Entwicklungsgang zu langen, wurmförmigen, grabenden und sich schlängelnden und daher gegliederten Tieren, anderseits $z u$ kriechenden, mit einem Kriechfuss versehenen Formen. Es drückt sich dieser Unterschied schon im Bau der Turbellarien-larve aus. Diese Larve ist entweder eine Protrochophora .(Protrochula), oder ein Proveliger; mit beiden zeigt sie Uebereinstimmung und beide lassen sịch leicht und ungezwungen auf sie zurückführen. Mit dem Proveliger zeigt die Turbellarien-larve Uebereinstimmung in der Metamorphose, weil sie sich zum erwachsenen Tier ausbaut; dies stimmt auch mit der Auffassung, dass Mollusken und Plathelminthen einander näher verwandt sind als Mollusken und Anneliden. Merkwürdig ist denn auch die Tatsache, dass, sobald bei den Plathelminthen die "Wurm"form erscheint, auch die Entwicklung mehr wurmähnlich wird. So bei den Nemertinen, welche die "Wurm"form erreicht und eine Pseudometamerie in Verband mit der, sei es auch schlaffen, aber doch gewissermassen schlängelnden Bewegung erlangt haben. Ihre Pilidiumlarve verhält sich denn auch prinzipiell wie die Trochophora; das junge Tier, so ganz verschieden vom Ausgangspunkt, entsteht nicht durch Verwandlung, sondern durch Einwachsen in die Larve und 
durch Neubildung (12, pag. 374). Die mit ihr verwandte Larvenform von Stilochus pilidium aber verwandelt sich wohl in das erwachsene Tier ohne die provisorische Larvenhüllen abzuwerfen. Es repräsentieren diese beiden Entwicklungstypen die beiden Richtungen, in welchen die Turbellarien sich entfaltet haben: einerseits die langen, segmentierten „Wurm"tiere, andrerseits die mehr compacten „Fuss”tiere mit kriechender Lebensweise. Diese meine Auffassung geht noch weiter, denn das Heer der "Trochophora"-ähnliche Larven ist ein grosses Heer; consequent durchgeführt müssen auch die pelagisch lebenden Cölenteraten - ich nehme an, dass diese unter den Cölenteraten die ursprünglichsten Typen repräsentieren - in diesem Kreise gehören; in der Tat halte ich auch diese Formen nicht für primitiv, sondern für durch Anpassung an das pelagische Leben ganz vereinfachte Lebewesen, welche u. A. ihr "mittleres Keimblatt” eingebüsst haben. Es ist hier aber nicht die Stelle näher auf diese Sache einzugehen.

\section{DIE CELL-LINEAGE}

Ein weiteres Argument für die enge Verwandtschaft zwischen Mollusken und Anneliden sucht HEIDER in den Resultaten, welche die Cell-lineage uns geliefert hat. Er schreibt: „Wir nehmen hier die Ergebnisse der neueren „Cell-Lineage"-Forschung kurz vorweg. Wenngleich den Turbellarien derselbe Spiraltypus der Zellklüftung zukommt wie den Anneliden und Mollusken, so ist doch zu erkennen, dass in Hinsicht auf die Furchungsbilder die beiden letzteren Gruppen einander näher stehen. Wir vermissen bei den Turbellarien die ungemein typische Figur des Kreuzes in der Episphäre, wie denn auch bei diesen Formen die in der Äquatorialzone gelegenen vier Gruppen von Trochoblasten nicht als differente Partien des Furchungsbildes zum Ausdruck kommen. Das erklärt sich aus dem Umstande, dass den Turbellarien ein typisch entwickeltes Trochophorastadium fehlt, dass sie auf der Vorstufe der Protrochula stehen bleiben. Als allgemeines Resultat der ontogenetischen Ueberprüfung der hier in Rede stehenden Gruppen können wir aussprechen, dass Mollusken und Anneliden die grösste Uebereinstimmung hinsichtlich ihres Entwicklungsverlaufes erkennen lassen, dass aber auch die Turbellarien diesem Entwicklungstypus einigermassen nahestehen" (8, pag. 508). Im Allgemeinen muss dies als richtig anerkannt werden; doch lässt sich auch einiges dagegen anführen. Erstens muss die Frage gestellt werden: gibt die Cell-lineage Andeutungen für die Lösung der Frage nach dem Zusammenhang zwischen den verschiedenen Typen? Hierüber kann man natürlich wieder verschieden urteilen; und die Würdigung wird viel Persönliches haben. Meines Erachtens aber wird der Wert der Celllineage, wie interessant ihre Kenntnis sonst sein mag, in dieser Hinsicht zu hoch angeschlagen. Wie man sich das Entstehen der mehrzelligen Tiere auch denkt und wie man sich diese ursprünglichen Lebewesen auch vorstellt, unmöglich können diese doch so gebaut gewesen sein, wie die Cell-lineage es in manchen Fällen logisch fordert. Stellt man sich ihr Entstehen vor als den Erfolg einer Kolonisierung, so haben doch sicher die Kolonisten denselben Wert, dieselbe Beschaffenheit und dieselben Potenzen; erst nach ihrem „Zusammenfliessen”, also viel später, ist eịne Arbeitsteilung eingetreten, welche natürlich sich nicht mit einem Male, sondern allmählich entwickelt hat. Denkt man an Zellfamilien, so könnte die Arbeitsteilung natürlich früher eintreten, vielleicht schon bei den ersten Teilungen. Ueberdies wird die Anordnung der Componenten solcher einfacher Kolonien oder Familien im ersten Anfang gewiss nicht regelmässig gewesen sein. Die Cell-lineage dagegen lehrt ganz andere Sachen. Vorerst kann die Determination der Zellen bei der Entwicklung der mehrzelligen Tiere sehr weit zurückgehen; schon bei den ersten Teilungen, ja selbst bei der ersten Teilung kann sie eintreten. Die Ontogenie recapituliert in dieser Hinsicht die Phylogenie gar nicht. Denn wenn bei der ersten Furchung bisweilen schon eine Teilung beobachtet wird, welche genau nach der Medianebene des späteren Tieres verläuft, so passt dies gewiss nicht in den Gedankengang, welchen wir uns von der phylogenetischen Entwicklung gemacht haben. Oder wenn z. B. bei Arenico! $a$ die Zelle $1 a^{2}$ im sechzehnzelligen Stadium schon als primärer Trochoblast angesehen werden kann, d. h. als die Mutterzelle, aus welcher sich die primären Trochoblasten $1 a^{211}, 1 a^{212}, 1 a^{221}$ und $1 a^{222}$ entwickeln, welche einen Teil der Anlage des praeoralen Wimperkranzes der Trochophora repräsentieren, so kann dies unmöglich ein ursprünglicher Zustand sein. Dasselbe gilt für die Anlage der apicalen Rosettenzellen, der Kreuz- und Intermediärzellen, welche alle schon im achtzelligen Stadium mit den primären Tro- 
choblasten in den Zellen $1 a-1 d$ potenziell vorhanden sind. So könnte man weitergehen. Die Anlagen sind zum grössten Teil als verfrüht, als zurückgeschoben anzusehen und haben mit der ursprünglichen Arbeitsteilung der Kolonie oder Familie nichts zu schaffen. Ein Bild der phylogenetischen Entfaltung von Merkmalen bietet uns die Cell-lineage gar nicht.

Dasselbe nun gilt auch für die verschiedenen Typen der Eiteilung selbst. Es ist nicht anzunehmen, dass der Radiär-, Bilateral-, Spiral- u.s. w. Typus schon bei den einfachst gebauten mehrzelligen Lebewesen sich vorgefunden hat; eine so gesetzmässig verlaufende Regelmässigkeit in der Stellung der Componenten ist undenkbar. Es bleibt natürlich hoch interessant, Gesetzen, welche die ersten Teilungen beherrschen, nachzuspüren; aber eine grosse Bedeutung für die Phylogenie können sie nicht besitzen. Es sei darauf hingewiesen, dass die Turbellarien - meines Erachtens mit den ursprünglich mehrzelligen Tieren, sei es auch natürlich in der Ferne, verwandt - in dieser Hinsicht die meist primitiven Zustände repräsentieren. Es kommt bei dieser Gruppe der Spiraltypus schon vor, z. B. bei Discocoelis. Daneben zeigen andere Formen ganz andere und mehr einfache Verhältnisse; so Planaria simplissima, bei welcher Art nach STEVENS die Blastomere unregelmässig angehäuft sind und Blastula und Gastrula vollkommen fehlen (18, pag. 217); weiter die Rhabdocoelen und Alloiocoelen, bei welchen Formen nach BRESSLAU die ersten Teilungsvorgänge fast regelmässig sich vollziehen, die weiteren Teilungen dagegen ganz unregelmässig verlaufen, sodass es völlig unmöglich wird, in den elf-, zwölf-, dreizehnoder mehrzelligen Stadien die Herkunft der einzelnen Blastomere mit Sicherheit zu ermitteln (2, pag. 229). Weiter kann bei den Rhabdocoelen weder von einer Gastrulation noch von Keimblättern gesprochen werden (2, pag. 322). Dasselbe fand MATtiesen bei den Süsswasserdendrocoelen (11, pag. 317 und 351). Ich bin geneigt, diese Zustände als primitiv anzusehen. Discocoelis ist in dieser Hinsicht weiter entwickelt; sie stimmt in ihrer Furchung mit Anneliden und Mollusken in vielen Hinsichten überein. Noch weiter geht Planocera nach den Untersuchungen von SuRfaCE (19), bei welcher Form die Teilung des dritten Ectomerenquartettes genau so stattfindet wie bei Anneliden und Mollusken, sodass auch hier die Zelle $4 d$ sich symmetrisch teilt in $4 d^{1}$ und $4 d^{2}$, welche beide Mesoderm liefern. Man findet also bei den Plathelminthen sowohl ganz primitive Zustände, wie solche, welche genau mit denen der genannten Gruppen übereinstimmen. Korschelt und HeIder sind denn auch geneigt, an Homologien zwischen den drei Gruppen in Bezug auf die Furchungsweise zu denken (10, pag. 69). SuRfaCE selbst schreibt hierüber: „In the spiral cleavage, the segregation of the ectoblast in three quartets, the formation of a large part of the mesoderm from $4 d$, the formation of the apical cells, and in many other details the development of the platodes corresponds to that of annelids and mollusks. These facts must tend to conform the view that in their early history these platodes were closely related to the two last mentioned phyla" (19, pag. 550). Und auch WILSON weist auf diese Uebereinstimmung hin; er schreibt: „The symmetrical division of this cell (nämlich die Zelle $4 d$ ) in the polyclade might accordingly be regarded as the prototype of that which occurs in the annelid or mollusk, though the resulting cell have in the latter forms a different morphological significance" (20, pag. 23). Es stehen also die Zustände bei Anneliden und Mollusken nicht isoliert einander gegenüber; aber eine Vermittelung durch die Plathelminthen ist logischer als die Annahme einer directen Verwandtschaft zwischen beiden Gruppen, welche direct auf die Plathelminthen zurückgeführt werden können.

Es lässt sich übrigens nicht verkennen, dass es in der ersten Entwicklung bei Anneliden und Mollusken sehr grosse Uebereinstimmung gibt. Daneben aber kommen auch sicher Unterschiede vor, auf welche Korschelt und HeIDER hinweisen. So liegen die Kreuzarme der Anneliden interradial, bei den Mollusken dagegen radial; sie verhalten sich zueinander wie Positiv und Negativ (10, pag. 101). Das Kreuz der Mollusken ist daher etwas anderes als das Kreuz der Anneliden (10, pag. 97). Bei den Anneliden sind die Zellen der Kreuzarme Abkommlingen der Zellen $1 a^{112}-1 d^{112}$, bei den Mollusken der Zellen $1 a^{12}-1 d^{12}$. Bei den Anneliden entsteht in den beiden Ventralarmen des Kreuzes je eine Doppelreihe von Zellen (Spaltung der Kreuzarme, 10, pag. 87); bei den Mollusken liegen am Ende der Kreuzarme die kleinen "tip cells" of the "cross" nach Conklin (10, pag. 98). Das Kreuz in der Entwicklung von Trochophora und Proveliger ist demnach nicht homolog, sondern wahrscheinlich eine analoge Bildung, welche mit der angepassten pelagischen Lebensweise zusammenhängt. Es liessen sich wahrscheinlich aus der Cell-lineage noch andere Unterschiede herausholen. Weil aber die Eier von Anneliden und 
Mollusken sich schnell zu einfach gebauten, pelagisch lebenden und an diese Lebensweise angepassten Larven entwickeln, so kann es nicht wundern, dass es viele Punkte von Uebereinstimmung gibt, wobei noch hinzukommt, dass beide Gruppen direct auf die Plathelminthen zurückgeführt werden können.

Alles in Allem finde ich nicht den geringsten Anlass, eine directe Verwandtschaft zwischen Anneliden und Mollusken anzunehmen. Ich glaube bei der Meinung, welche früher von mir verteidigt worden ist, dass es in der Tat einen näheren Zusammenhang zwischen Mollusken und Plathelminthen gibt als zwischen Mollusken und Anneliden, bleiben zu müssen. Die Anhänger dieser Meinung Lang, Plate, Thiele und Simroth -- sind noch verstärkt worden durch OdHner, welcher in seinen letzten interessanten Studien über Solenogastren von Norwegen zu demselben Resultat gekommen ist (14). Der Rahmen dieses Artikels lässt aber nicht zu, näher hierauf und auch auf die Untersuchungen ODHNER's, welche wichtige Anknüpfungspunkte bieten, einzugehen; hoffentlich werde ich später Gelegenheit finden, auch auf diese Sachen zurückzukommen.

\section{LITERATURLISTE}

1. O. ABEL, Palaeobiologie der Cephalopoden aus der Gruppe der Dibranchiaten, Jena, Fischer, 1916.

2. E. BREsSlau, Beiträge zur Entwicklungsgeschichte der Turbellarien. I. Die Entwicklung der Rhabdocölen und Alloiocölen. Zeitschr. f. Wiss. Zool. Vol. 76, 1904, pag. 213-332.

3. C. Clifford Dobell, The Principles of Protistology. Archiv f. Protistenkunde, Vol. 23, 1911, pag. $269-310$.

4. F. Doflein, Lehrbuch der Protozoenkunde, 4te Auflage, Jena, Fischer, 1916.

5. M. Hartmann, Protozoa, Handwörterbuch d. Naturwiss. Vol. 7, Jena, Fischer, 1912, pag, 1126-1135.

6. - Allgemeine Morphologie und Physiologie der Protozoen. Lehrbuch d. Mikrobiologie, Vol. 1 (Allgemeiner Teil), Jena, Fischer, 1919, pag. 89-130.

7. K. HeIDER, Entwicklungsgeschichte und Morphologie der Wirbellosen. - Die Kultur der Gegenwart, Teil 3, Abteilung 4, Band 2; Zellen und Gewebelehre, Morphologie und Entwicklungsgeschichte, II, Zoologischer Teil; Leipzig-Berlin, Teubner, 1913, pag. 176-332.

8. - - Phylogenie der Wirbellosen. - Die Kultur der Gegenwart, Teil 3, Abteilung 4, Band 4; Abstammungslehre, Systematik, Palaeontologie, Biogeographie; Leipzig-Berlin, Teubner, 1914, pag. 453-529.

9. E. KorSchelT, Lebensdauer, Alter und Tod. Jena, Fischer, 1922.

10. - - und K. HEIDER, Lehrbuch der Vergleichenden Entwicklungsgeschichte der wirbellosen Tiere. Allgemeiner Teil, Lief. 3., Jena, Fischer, 1909.

11. E. Mattiesen, Ein Beitrag zur Embryologie der Süsswasserdendrocölen. Zeitschr. f. Wiss. Zool., Vol. 77, 1904, pag. 274-361.

12. A. NAEF, Studien zur generellen Morphologie der Mollusken. II. Das Cölomsystem in seinen topographischen Beziehungen. Ergebn. u. Fortschr. d. Zoologie, Vol. 3, 1913, pag. 329-462.

13. H. F. Nierstrasz, Das Herz der Solenogastren. Verhandl. Kon. Akad. v. Wetensch. Amsterdam, Sect. 2; Vol. 10, $\mathrm{n}^{\circ}$ 2, 1903, pag. $1-52$.

14. NiLs HJ. ODHNER, Norwegian Solenogastres. Bergens Museums Aarbok 1918-19, Naturvid. raekke $\mathrm{n}^{\circ} 3$, pag. 1-86.

15. P. Pelseneer, Les Lamellibranches de l'Expédition du Siboga. Siboga-Exped., Uitkomsten op Zoölogisch, Botanisch, Oceanographisch en Geologisch Gebied, Leiden, Brill, Monogr. 53a, 1911.

16. J. W. SPEngel, Sipunculidea. Handwörterbuch d. Naturwiss. Jena, Fischer, Vol. 9, 1913, pag. 96-106.

17. G. STEINER, Untersuchungen über den allgemeinen Bauplan des Nematodenkörpers. Zool. Jahrb., Anat. u. Ontog., Vol. 43, 1921, pag. 1-96.

18. N. M. Stevens, On the Germ Cells and the Embryology of Planaria simplissima. Proceed. Acad. Natur. Sc. Philadelphia, Vol. 56, 1904-1905, pag. 208-220.

19. Frank M. Surface, The Early Development of a Polyclad, Planocera inquilina Wh. Proceed. Acad. Natur. Sc. Philadelphia, Vol. 59, 1907-1908, pag. 514-559.

20. E. B. Wilson, Considerations on Cell-Lineage and Ancestral Reminiscence, based on a Re-examination of Some Points in the Early Development of Annelids and Polyclades. Annals New York Acad. Sc., Vol. 11, 1898, pag. 1-27.

21. R. Woltereck, Wurm,kopf”, Wurmrumpf und Trochophora. Zool. Anz. Vol. 28, 1905, pag. $273-322$. 